\title{
Grau de Legibilidade do Novo Relatório do Auditor Independente
}

\author{
Level of Readability in the New Report of the Independent Auditor
}

Donizete Reina ${ }^{1}$, Filipe Augusto Sousa e Silva², Sirlei Lemes², Diane Rossi Maximiano Reina ${ }^{1}$

'Universidade Federal do Espírito Santo, Espírito Santo, Brasil. ${ }^{2}$ Universidade Federal de Uberlândia, Minas Gerais, Brasil.

I N F O A R T I GO

\section{Palavras-chave:}

Complexidade

Informacional,

Legibilidade,

Relatório do Auditor

Independente,

Índice Brasil 100 (IBrX

100).

A R T I C LE I N F O

Keywords:

Informational Complexity,

Readability,

Report of the Independent

Auditor,

Brazil 100 Index (IBrX 100).

\begin{abstract}
RESUMO
Este artigo tem como objetivo analisar se o grau de legibilidade dos relatórios do auditor independente foi afetado após as mudanças das normas de elaboração desses relatórios. Em vista desse objetivo, foram analisados 273 relatórios de auditor independente, divulgados entre os anos de 2015 e 2017, em empresas listadas no Índice Brasil 100 (IBrX 100). A mensuração da legibilidade foi realizada pelo Gunning's Fog Index ("índice de nevoeiro"), programa que permite identificar o grau de complexidade desses relatórios, em conjunto com o teste de correlação estatística. Os resultados apontaram que, na média, houve uma redução de $48 \%$ para $40 \%$ no grau de complexidade, tanto no ano de 2016 como em 2017. A correlação foi positiva e com magnitude moderada entre as variáveis grau de legibilidade e nível de governança corporativa. Por fim, dentre os relatórios analisados, o da empresa Light S/A foi o que apresentou o menor grau de complexidade de todas as empresas relacionadas neste estudo, no ano de 2015. Por sua vez, o da empresa B2W Digital foi o que apresentou o maior grau de complexidade no mesmo ano, com grau de $147 \%$.

A B S T R A C T

This paper aims to analyze whether the degree of readability of the independent auditor's reports was affected after the changes in the rules for preparing these reports. In view of this objective, 273 independent auditor's reports, published between the years 2015 and 2017, in companies listed in the Brazil 100 Index (IBrX 100) were analyzed. The readability measurement was performed using the Gunning's Fog Index ("fog index"), a program that allows the identification of the degree of complexity of these reports, together with the statistical correlation test. The results showed that, on average, there was a reduction from $48 \%$ to $40 \%$ in the degree of complexity, both in 2016 and in 2017. The correlation was positive and with moderate magnitude between the variables degree of readability and level of corporate governance. Finally, among the analyzed reports, the one by the company Light S/A was the one with the lowest degree of complexity of all the companies listed in this study, in 2015. In turn, the one by the company B2W Digital was the one that presented the highest degree of complexity in the same year, with a degree of $147 \%$.
\end{abstract}




\author{
Correspondência para autores: \\ donizete.reina@ufes.br (Reina, D.) (ORCID: 0000-0001-6217-2324), \\ filipea133@gmail.com (Silva, F.A.S.) (ORCID: 0000-0002-8101-8282), \\ sirlemes@uol.com.br (Lemes, S.) (ORCID: 0000-0003-3334-4240), \\ dianereina@hotmail.com (Reina, D.R.M.) (ORCID: 0000-0001-9683-9991).
}

\title{
1. Introdução
}

O novo Relatório do Auditor Independente traz consigo as novas regras e novas exigências, fazendo com que o grau de confiança nas informações da empresa aumente, ao trazer maior clareza para o investidor no momento de escolher seus investimentos. O êxito das transações no mercado de capitais está relacionado à apresentação de boas informações ao investidor na hora de tomar decisões com mais confiança e menos perigo (PATROCINIO et al., 2017). Assim, ao versar sobre as vantagens e desvantagens para as empresas do mercado de capitais e as dificuldades identificadas pelo auditor independente, quando da realização de suas atividades de auditoria e seus respectivos relatórios de auditoria, as alterações das Normas Brasileiras de Contabilidade e Auditoria Independente - NBC 260, 570, 700, 701, 705, 706 (2016) têm como objetivos melhorar a eficiência na divulgação dos relatórios do auditor.

De acordo com Souza et al. (2019), os gerentes podem, intencionalmente, reduzir a legibilidade das divulgações contábeis narrativas, tornando-as mais complexas. Tal suposição é, teoricamente, chamada de hipótese de ofuscação gerencial. Nessa perspectiva, Miranda, Reina e Lemes (2018) destacam que a legibilidade pode contribuir para que os usuários alcancem uma melhor interpretação e análise das informações e dos dados contidos nos relatórios financeiros. A legibilidade poderia, ainda, se tornar um indicador de desempenho das informações escritas e da eficácia dos instrumentos de comunicação financeiros, aliada a outras formas de mensuração do diclosure e, consequentemente, do grau de legibilidade das informações que estão sendo apresentadas pelas empresas (MIRANDA; REINA; LEMES, 2018).

As Normas Brasileiras de Contabilidade (NBC) constituem um conjunto de regras a serem seguidas para o exercício da profissão contábil, como uma atividade, cujo fim é atestar a situação das operações das empresas, com a intenção de gerar confiabilidade (NBC 260, 570, 700, 701, 705, 706, 2016). O novo relatório, por sua vez, traz maior responsabilidade para o auditor, tendo em vista que a opinião dele ganhou destaque, constituindo o primeiro parágrafo do relatório e sendo apresentada como: limpa, com ressalva, adversa ou, quando necessário, o auditor pode se abster de opinar. Dessa forma, com as alterações nas normas, o auditor pode apresentar informações mais claras, objetivas e mais abrangentes, facilitando a compreensão e aumentando a importância do relatório para a tomada de decisão dos usuários internos e externos, seguindo as normas - NBC 260, 570, 700, 701, 705, 706 (2016).

A auditoria externa é considerada um importante mecanismo de controle tanto para a empresa que é auditada, quanto para a comunidade externa à empresa, que podem utilizar as informações para possíveis investimentos, tendo em vista que o relatório da auditoria externa apresenta dados relevantes da companhia auditada, inclusive, podendo apontar equívocos provocados involuntariamente, ou, ainda, fraudes que possam ter acontecido devido a atos ilícitos e de má fé. Para Souza et al. (2019), a demanda por informações contábeis e financeiras requer melhor e mais profundo entendimento da motivação em torno da elaboração desses 
relatórios, pois, por meio da ofuscação gerencial, as descrições contábeis narrativas podem ser elaboradas de forma dificultar a extração da informação.

Pelo exposto até aqui, busca-se, neste estudo, preencher uma lacuna de pesquisa identificada e que levou à seguinte pergunta: as novas normas do Relatório do Auditor Independente afetaram o grau de legibilidade dos relatórios de auditoria das empresas listadas no Índice Brasil 100 (IBrX 100), tornando-os mais complexos? Para responder à pergunta de pesquisa e preencher tal lacuna, buscou-se, então, analisar se o grau de legibilidade dos relatórios do auditor independente foi afetado após as mudanças das normas de elaboração desses relatórios.

Desse modo, a contribuição desta pesquisa decorre, justamente, por verificar a legibilidade dos relatórios de auditoria pós-adoção do IFRS, constituindo um diferencial entre as pesquisas sobre a temática da legibilidade. Este estudo, por fim, sustenta sua contribuição, observando a afirmação de Ricci (2014), de que os relatórios de auditoria apresentam informações levantadas com finalidade de promover a comunicação clara entre o auditor e o leitor das demonstrações financeiras, visto que podem influenciar na tomada de decisão dos investidores e demais usuários de informações contábeis.

Nesse sentido, ainda, este estudo contribui para o debate acadêmico sobre como a legibilidade narrativa dos relatórios de auditoria pode afetar tanto a compreensão destes quanto a sua forma de elaboração e divulgação. Assim, assume-se que informações com maior legibilidade são mais confiáveis e menos enganosas, e que a legibilidade da informação contábil, especificamente do relatório do auditor, está relacionada ao grau de complexidade com que essas informações são apresentadas ao mercado. Em razão disso, ressalta-se a necessidade de investigar se a norma internacional (por meio das normas do novo relatório do auditor), com todos os seus requisitos, está influenciando na legibilidade desses relatórios.

\section{Fundamentação teórica}

\subsection{Relatório do auditor independente e sua legibilidade}

Segundo Baker e Kare (1992), os relatórios financeiros anuais obrigatórios constituem os meios convencionais de comunicação entre os gerentes de uma empresa e os seus acionistas. Contudo, esses relatórios financeiros precisam atender também a outras partes interessadas da empresa, nomeadamente os funcionários, fornecedores, clientes, a comunidade de investidores e a sociedade em geral (BAKER; KARE, 1992). Por meio dos relatórios financeiros anuais, a gestão pode disseminar informações que reduzem a incerteza sobre a empresa por parte dos acionistas, além de assegurar aos funcionários, aos detentores de títulos e aos fornecedores que a participação econômica na empresa é segura (BAKER; KARE, 1992).

Com as alterações nas IFRS, os relatórios de auditoria independente também foram alterados. No Brasil, essas modificações foram previstas nas Normas Brasileiras de Contabilidade Técnica de Auditoria (NBC TA) e entraram em vigor as auditorias das demonstrações contábeis para periódicos que se encerraram em ou após 31 de dezembro de 2016. Segundo a NBC TA 570 (2016) - Continuidade Operacional, republicada em 2016, trazendo uma nova redação, o auditor, além da responsabilidade de obter evidências de auditoria de que a administração da empresa, ao realizar suas demonstrações contábeis, está utilizando a base contábil de continuidade operacional deve, ainda, relatar se houver incerteza significativa da capacidade da empresa em continuar com suas atividades. 
Outra alteração é observada na NBC TA 700 (2016) - Formação da Opinião e Emissão do Relatório do Auditor Independente, exigindo do auditor que, ao formar sua opinião referente às demonstrações contábeis, expresse-a clara e objetivamente, e a coloque no início do relatório, facilitando aos usuários da informação identificarem qual tipo de opinião ele expressou e quais bases adotou para expressá-la.

A NBC TA 701(2016) - Comunicação dos Principais Assuntos de Auditoria no Relatório do Auditor Independente - dispõe sobre a responsabilidade do auditor de comunicar os principais assuntos de auditoria no relatório, deixando-o mais informativo. $\mathrm{O}$ assunto é colocado logo na sequência da opinião, com o objetivo de destacar essas informações, tendo em vista que são elas, segundo o julgamento do auditor, que impactam direta e expressivamente nos resultados da empresa.

Em relação à opinião propriamente dita, a NBC TA 705 (2016) - Modificações na Opinião do Auditor Independente - orienta que as opiniões podem se apresentar, como: opinião limpa, quando não há evidência de auditoria suficiente, e as demonstrações representam adequadamente a realidade da empresa; opinião com ressalva, quando, ao realizar a auditoria, o auditor se depara com uma distorção relevante, porém não generalizada; opinião adversa, que deve aparecer no relatório quando as distorções encontradas são relevantes e generalizadas, levando o auditor a especificar que, em sua opinião, as demonstrações não representam a posição patrimonial e financeira da entidade; o auditor pode se abster de opinar, ou seja, ele pode especificar que não expressa opinião, quando não encontra evidências suficientes para formar opinião e conclui que as possíveis distorções seriam relevantes e generalizadas.

A NBC TA 706 (2016) - Parágrafos de Outros Assuntos permite ao auditor chamar a atenção dos usuários, quando julgar necessário, através da menção de algum evento relevante, já divulgado e revelado em notas explicativas. No Quadro 1, a seguir, podem-se verificar os principais pontos de alteração dessas cinco NBC TA.

Quadro 1 - Principais alterações no relatório do auditor independente.

\begin{tabular}{|c|l|}
\hline \multicolumn{2}{|c|}{ Quadro Resumo } \\
\hline $\begin{array}{c}\text { Relatório do Auditor } \\
\text { Independente }\end{array}$ & $\begin{array}{l}\text { O Relatório do Auditor Independente é uma atividade que tem por fim atestar a } \\
\text { situação das operações das empresas com a intenção de gerar confiabilidade. }\end{array}$ \\
\hline $\begin{array}{c}\text { Normas Brasileiras de } \\
\text { Contabilidade }\end{array}$ & $\begin{array}{l}\text { As Normas Brasileiras de Contabilidade (NBC) constituem um conjunto de regras } \\
\text { a serem seguidas para o exercício da profissão contábil. }\end{array}$ \\
\hline \multirow{2}{*}{ NBC TA 570 } & $\begin{array}{l}\text { Trata da responsabilidade do auditor de encontrar evidências suficientes para } \\
\text { comprovar que a empresa utiliza a base contábil de continuidade operacional. } \\
\text { Vigência: a partir de 31 de dezembro de 2016. }\end{array}$ \\
\hline NBC TA 700 & $\begin{array}{l}\text { Dispõe sobre a opinião do auditor, que passa a ser publicada no início do relatório, } \\
\text { devendo ser clara e objetiva. Vigência: a partir de 31 de dezembro de 2016. }\end{array}$ \\
\hline NBC TA 701 & $\begin{array}{l}\text { Dispõe sobre a responsabilidade do auditor de comunicar os principais assuntos de } \\
\text { auditoria no relatório, logo após a opinião, deixando-o mais informativo. Vigência: } \\
\text { a partir de 31 de dezembro de 2016. }\end{array}$ \\
\hline NBC TA 705 & $\begin{array}{l}\text { Trata das modificações que a opinião do auditor pode apresentar, sendo: opinião } \\
\text { limpa, com ressalva ou adversa; o auditor pode também se abster de opinar. } \\
\text { Vigência: a partir de 31 de dezembro de 2016. }\end{array}$ \\
\hline
\end{tabular}




\begin{tabular}{|c|l|}
\hline \multirow{2}{*}{ NBC TA 706 } & $\begin{array}{l}\text { Permite ao auditor escrever Parágrafos de Outros Assuntos de Auditoria no } \\
\text { relatório para chamar a atenção para fatos que, segundo seu julgamento, sejam } \\
\text { relevantes. Vigência: a partir de 31 de dezembro de 2016. }\end{array}$ \\
\hline \multirow{2}{*}{$\begin{array}{c}\text { Novo Relatório do } \\
\text { Auditor Independente }\end{array}$} & $\begin{array}{l}\text { As alterações nas normas permitem que o auditor apresente informações, mais } \\
\text { claras e objetivas, facilitando a compreensão e aumentando a importância do } \\
\text { relatório no momento da tomada de decisão pelos usuários internos e externos. }\end{array}$ \\
\hline
\end{tabular}

Fonte: Elaborado pelos autores, a partir das NBC TA 570, 700, 701, 705, 706 (2016).

Estudos revelam algumas características envolvendo o nível de legibilidade dos relatórios financeiros, como: impactar, negativamente, o princípio da comunicação eficaz, quando enviesado (COURTIS, 1986); resultados que podem ser gerenciados para deixar as informações divulgadas menos legíveis (LI, 2008); por vezes, a linguagem de comunicação mais complexa é adotada para confundir os usuários (RENNEKAMP, 2012); informações mais complexas são divulgadas e ofuscadas sob a égide da legalidade e tecnicalidade, próprias dessas informações divulgadas (LO; RAMOS; ROGO, 2017); relatórios com informações mais complexas prejudicam sua usabilidade (LEHAVY; LI; MERKLEY, 2011); e, se informações mais complexas podem prejudicar o grau de entendimento dos usuários em função de relatórios distorcidos, então, é necessário divulgar informações menos complexas e que atinjam seu objetivo (MIRANDA; REINA; LEMES, 2018); pois, uma informação financeira compreensível pode favorecer a avaliação do investidor, despertando-lhe mais confiança na administração da entidade, agregando credibilidade a ela (RENNEKAMP, 2012).

Segundo Courtis (1995), um dos princípios da comunicação efetiva compreende que as mensagens recebidas pelos leitores sejam interpretadas da mesma forma que o pretendido pelo remetente. Ademais, no relatório corporativo, a narrativa textual representa a maior parte da divulgação, sendo, em média, $80 \%$ de um relatório anual em relação ao restante, que consiste em números e representação de dados quantitativos (LO; RAMOS; ROGO, 2017).

Por fim, o estudo de Rennekamp (2012) destaca as medidas tomadas pela SEC (Securitie and exchange comssion), para desenvolver maior legibilidade nos relatórios financeiros, utilizados por investidores de empresas americanas. Outras pesquisas, como: Miller (2010), Courtis (1986), Courtis (1995), Lehavy, Li e Merkley (2011), Curto (2014), Fakhfakh (2015), Buschee, Gow e Taylor (2018), Miranda, Reina e Lemes (2018), e Silva, Lemes e Reina (2020), testaram a legibilidade das informações por meio do grau de complexidade destas.

\subsection{Estudos Anteriores}

De acordo com Li (2008), o maior grau de complexidade na leitura dos relatórios das companhias está associado a divulgações relacionadas a ganhos menores, assim como, a menores investimentos feitos pelas empresas. No estudo de Li (2008), são identificadas as primeiras evidências sobre as implicações lexicais nas divulgações corporativas e a legibilidade do relatório anual. Os resultados apontaram que os relatórios anuais de empresas com desempenho fraco são mais difíceis de ler; já, quando o lucro das empresas é mais persistente, os relatórios são mais fáceis de ler. Outro resultado apontou que a alteração da legibilidade anual tem, aproximadamente, o mesmo impacto na persistência de lucros, como as provisões, sugerindo que 
os gerentes podem estruturar, oportunisticamente, o relatório anual para esconder, dos investidores, informações adversas. Nesse sentido, segundo alertam Silva e Fernandes (2009), é preciso ter atenção especial para a maneira como os textos narrativos dos fatos relevantes são divulgados, haja vista que a legibilidade pode representar melhoria, ao evidenciar a informação contábil.

O estudo de Miller (2010), sobre o efeito que a complexibilidade da informação financeira causa nos investidores, revelou que o tamanho e a legibilidade dos relatórios financeiros influenciam no comportamento, tanto dos pequenos quanto dos grandes investidores. Além disso, mesmo que evidências experimentais sugiram que os pequenos investidores sejam mais afetados pelos atributos dos relatórios financeiros, outras evidências mostram que, mesmo os maiores e sofisticados investidores, sofrem influência da complexidade da informação (MILLER, 2010).

A legibilidade é um critério indispensável para se elaborar qualquer tipo de informação. Dentro da contabilidade e do mercado financeiro, este é um fator de extrema importância, dada a relevância da compreensão dos variados tipos de relatórios financeiros para os investidores ou àqueles interessados na informação (PORTO et al., 2014). A legibilidade pode ser conceituada como a capacidade de se compreender e interpretar determinada leitura, que se destaca num texto claro e nítido, isto é, de fácil entendimento, destacando-se que um conteúdo textual com frases curtas facilita maior legibilidade, dado que apresentam menor quantidade de palavras e caracteres (PORTO et al., 2014).

Observa-se que os estudos anteriores sobre a legibilidade dos relatórios anuais, os quais baseavamse, principalmente, nas narrativas inglesas, identificaram que os relatórios são, deveras, difíceis de ler. Moreno e Casasola (2016), por exemplo, testaram diversas variáveis que podem influenciar a legibilidade, incluindo rentabilidade. Os resultados, embora indiquem uma melhoria na legibilidade ao longo dos anos, confirmaram que os relatórios são, de fato, de difícil leitura. Nesse sentido, mesmo concluindo que as narrativas são difíceis de ler, ressalta-se que, por longo período, uma série de variáveis contextuais, como diferentes regimes políticos, um aumento do nível da cultura da população ou mesmo a evolução da própria linguagem, podem afetar a evolução de relatórios anuais (MORENO; CASASOLA, 2016).

Segundo o estudo de Buschee, Gow e Taylor (2017), a complexidade de informação em conference calls pode estar ligada tanto ao excesso de informações técnicas quanto à ofuscação de informações. Os resultados mostraram que, quando há maior complexidade textual nas amostras analisadas, tanto o excesso de informações técnicas quanto a ofuscação de dados importantes são culpados na mesma medida, ou seja, este maior grau de legibilidade pode estar relacionado a ambos os problemas.

O estudo de Lo, Ramos e Rogo (2017), por sua vez demostrou resultados distintos aos identificados por Li (2008), apresentados no início deste tópico, apontando evidenciando que empresas que se mostraram mais lucrativas em seus relatórios contábeis possuíam maior índice de legibilidade (FOG) em seus MD\&As (Management Discussion and Analysis), uma porção relevante de seus relatórios anuais.

Estudos em nível nacional, como o de Santos, Calixto e Bispo (2018), procedendo à análise de empresas brasileiras não financeiras, listadas na $[\mathrm{B}]^{3}$ Brasil, Bolsa, Balcão, observaram que o impacto da OCPC 07, no enxugamento de Notas Explicativas (NE), não corroborou a diminuição do nível de legibilidades dessas notas, mostrando esse índice como independente do tamanho dos relatórios.

Souza et al. (2019) investigaram a relação entre a legibilidade das narrativas dos relatórios 
financeiros e o desempenho financeiro, observando o argumento de que os gerentes elaboram uma contabilidade narrativa mais complexa para divulgação quando o desempenho financeiro é ruim. Os resultados apontaram que os gerentes, deliberadamente, acrescentam complexidade às divulgações contábeis narrativas para ocultar informações sobre o desempenho ruim; assim como, que informações complexas passadas têm impacto negativo no desempenho atual das empresas e que narrativas complexas são usadas como subterfúgio por ocultar informações financeiras negativas.

Por fim, em estudo mais recente, Silva, Lemes e Reina (2020) analisaram o efeito da adoção da OCPC 07 na legibilidade das informações divulgadas nas notas explicativas (NE) e a correlação entre o número de páginas divulgadas e o grau de complexidade dessas notas. Os resultados mostraram que, no ano de 2016, houve uma pequena redução do índice de legibilidade das NE em relação ao ano de 2015. Contudo, apesar dessa leve redução, os índices de legibilidade calculados estavam distantes dos 10 pontos considerados como ideais pelo teste de GFI, sugerindo que apenas a nova orientação não foi suficiente para aumentar a legibilidade das NE.

\section{Metodologia da Pesquisa}

Para este estudo, o índice de legibilidade dos relatórios do auditor independente foi extraído por meio do programa "Gunning's Fog Index", representado a seguir (Equação 1). Dos 100 papéis mais negociados, listados no Índice Brasil 100 (IBrX 100), foram identificadas 96 empresas. Dessa amostra inicial, as empresas Azul, Petrobras Br, Carrefur, Ir Brasil, e Smiles não disponibilizaram seus relatórios de auditoria, perfazendose uma amostra final de 91 empresas. Ao todo, foram analisados 273 relatórios de auditoria independente, realizados ao longo dos anos de 2015 a 2017. Os dados foram coletados nos sites das companhias e no site da $[B]^{3}$ Brasil, Bolsa, Balcão.

$$
\mathrm{GFI}=\left[\left(\frac{\text { número de palavras }}{\text { número de frases }}\right)+(\text { número de palavras difíceis })\right] \cdot 0,4
$$

O objetivo desse programa de legibilidade é determinar o quão difícil é ler e entender determinada informação divulgada, em que "palavras difíceis" representam as palavras complexas, escritas no decorrer do texto (GUNNING, 1952). Ainda segundo Gunning (1952), quanto maior (menor) a classificação de acordo com o índice, mais (menos) complexa é a leitura do texto. Assim, o FOG Index testa a legibilidade do texto e calcula a média ponderada do número de palavras por frase, bem como o número de palavras longas (três ou mais sílabas), gerando um índice com essas observações agrupadas (GUNNING, 1952). Esse índice também é conhecido como "índice de névoa", considerando a relação direta que tem com o grau de compreensibilidade das informações apresentadas pelas empresas. A interpretação adequada da escrita profissional deve apresentar um índice entre 10 e 15. Assim, um índice maior que 15 indica que o texto apresenta uma linguagem complexa, e um índice abaixo de 10 aponta para um texto muito simplificado (GUNNING, 1952).

\section{Apresentação dos Resultados}

O período de análise compreendeu os anos de 2015-2017, visando abranger um ano (2015) antes da adoção da norma; o ano da adoção (2016); e um ano (2017) após a adoção da norma. Sobre a avaliação da qualidade de um texto, Curto (2014) afirma que ela é baseada na estrutura, na escrita e na facilidade de sua leitura. Para Fakhfakh (2015), a compreensão está relacionada, linguisticamente, à percepção do signifícado 
correto do texto, assim como à inteligibilidade, que deve possibilitar aos leitores ler determinado conteúdo da informação e captar o significado das ideias apresentadas. A Tabela 1, apresentada no próximo tópico, foi elaborada, para fazer constar a relação das empresas (em ordem alfabética) que serviram de base para este estudo, assim como os índices de legibilidade dos seus respectivos pareceres.

Tabela 1 - Relação das empresas e respectivos índices FOG Index identificados.

\begin{tabular}{|c|c|c|c|c|c|c|c|c|c|}
\hline Empresa & 2015 & Parecer & Auditoria & 2016 & Parecer & Auditoria & 2017 & Parecer & Auditoria \\
\hline AMBEV & 30.0 & S. R. & Deloitte & 28.9 & S. R. & Deloitte & 27.8 & S. R. & Deloitte \\
\hline ALUPAR & 28.9 & S. R. & KPMG & 30.5 & S. R. & KPMG & 29.9 & S. R. & KPMG \\
\hline AREZZO CO & 28.4 & S. R. & EY & 28.6 & S. R. & PWC & 28.7 & S. R. & PWC \\
\hline B3 & 28.4 & S. R. & EY & 27.7 & S. R. & EY & 26.5 & S. R. & EY \\
\hline BANCO BRASIL & 27.2 & S. R. & KPMG & 29.5 & S. R. & KPMG & 29.6 & S. R. & KPMG \\
\hline BRADESCO & 27.3 & S. R. & KPMG & 28.2 & S. R. & KPMG & 28.3 & S. R. & KPMG \\
\hline BBSEGURIDADE & 28.6 & S. R. & KPMG & 30.4 & S. R. & KPMG & 30.4 & S. R. & KPMG \\
\hline MINERVA & 28.3 & S. R. & BDO BR & 27.9 & S. R. & $\begin{array}{c}\text { Grant } \\
\text { Thornton } \\
\text { Br. }\end{array}$ & 29.6 & S. R. & $\begin{array}{c}\text { Grant } \\
\text { Thornton } \\
\text { Br. }\end{array}$ \\
\hline BRADESPAR & 29.7 & S. R. & KPMG & 29.6 & S. R. & KPMG & 30.3 & S. R. & KPMG \\
\hline BRF AS & 28.1 & S. R. & EY & 28.2 & S. R. & EY & 30.0 & S. R. & KPMG \\
\hline BRASKEM & 29.3 & S. R. & KPMG & 29.8 & S. R. & KPMG & 30.3 & S. R. & KPMG \\
\hline BR MALLS PAR & 26.0 & S. R. & EY & 29.7 & S. R. & EY & 28.2 & S. R. & PWC \\
\hline BANRISUL & 26.7 & S. R. & EY & 29.5 & S. R. & KPMG & 30.2 & S. R. & KPMG \\
\hline B2W DIGITAL & 37.1 & S. R. & PWC & 32.6 & S. R. & KPMG & 31.1 & S. R. & KPMG \\
\hline CCR AS & 28.5 & S. R. & Deloitte & 31.2 & S. R. & Deloitte & 31.7 & S. R. & KPMG \\
\hline CESP & 31.4 & S. R. & EY & 28.1 & S. R. & EY & 28.1 & S. R. & BDO BR \\
\hline CIELO & 29.2 & S. R. & KPMG & 31.2 & S. R. & KPMG & 32.1 & S. R. & KPMG \\
\hline CEMIG & 25.5 & S. R. & Deloitte & 27.6 & S. R. & Deloitte & 28.3 & S. R. & EY \\
\hline CPFL ENERGIA & 30.0 & S. R. & Deloitte & 32.1 & S. R. & Deloitte & 30.0 & S. R. & KPMG \\
\hline COPEL & 36.4 & S. R. & KPMG & 30.0 & S. R. & Deloitte & 29.7 & S. R. & Deloitte \\
\hline COSAN & 30.8 & S. R. & KPMG & 29.6 & S. R. & KPMG & 30.8 & S. R. & KPMG \\
\hline COPASA & 34.2 & C. R. & PWC & 28.7 & C. R. & PWC & 29.4 & S. R. & PWC \\
\hline SID NACIONAL & 30.1 & S. R. & Deloitte & 29.4 & S. R. & Deloitte & 29.3 & S. R. & $\begin{array}{c}\text { Grant } \\
\text { Thornton } \\
\text { Br. }\end{array}$ \\
\hline CVC BRASIL & 28.9 & S. R. & EY & 27.7 & S. R. & $\mathrm{EY}$ & 28.1 & S. R. & $\mathrm{EY}$ \\
\hline CYRELA REALT & 31.3 & S. R. & Deloitte & 29.4 & S. R. & Deloitte & 29.8 & S. R. & KPMG \\
\hline DURATEX & 28.1 & S. R. & EY & 28.6 & S. R. & EY & 30.1 & S. R. & $\mathrm{EY}$ \\
\hline ECORODOVIAS & 27.9 & S. R. & $\mathrm{EY}$ & 27.1 & S. R. & EY & 28.3 & S. R. & EY \\
\hline ENGIE BRASIL & 30.1 & S. R. & KPMG & 30.0 & S. R. & KPMG & 28.8 & S. R. & Deloitte \\
\hline
\end{tabular}




\begin{tabular}{|c|c|c|c|c|c|c|c|c|c|}
\hline ELETROBRAS & 28.3 & S. R. & KPMG & 28.1 & S. R. & KPMG & 30.0 & S. R. & KPMG \\
\hline ELETROPAULO & 27.6 & S. R. & EY & 27.6 & S. R. & EY & 28.2 & S. R. & EY \\
\hline EMBRAER & 28.9 & S. R. & KPMG & 31.6 & S. R. & KPMG & 28.3 & S. R. & PWC \\
\hline ENERGIAS BR & 27.3 & S. R. & PWC & 28.2 & S. R. & PWC & 28.7 & S. R. & PWC \\
\hline ENERGISA & 27.0 & C. R. & Deloitte & 28.8 & S. R. & Deloitte & 31.2 & S. R. & $\mathrm{EY}$ \\
\hline EQUATORIAL & 29.4 & S. R. & $\mathrm{EY}$ & 30.7 & S. R. & KPMG & 30.1 & S. R. & KPMG \\
\hline ESTACIO PART & 31.6 & S. R. & PWC & 29.8 & S. R. & PWC & 29.0 & S. R. & EY \\
\hline EZTEC & 30.6 & S. R. & Deloitte & 29.7 & S. R. & Deloitte & 30.5 & S. R. & Deloitte \\
\hline FIBRIA & 28.6 & S. R. & Baker Tilly & 30.9 & S. R. & BDO BR & 29.0 & S. R. & BDO BR \\
\hline FLEURY & 26.6 & S. R. & PWC & 30.9 & S. R. & KPMG & 31.2 & S. R. & KPMG \\
\hline GERDAU & 27.5 & S. R. & PWC & 29.4 & S. R. & PWC & 31.5 & S. R. & KPMG \\
\hline GERDAU MET & 27.3 & S. R. & PWC & 28.4 & S. R. & PWC & 31.8 & S. R. & KPMG \\
\hline GOL & 27.6 & S. R. & EY & 28.0 & S. R. & EY & 28.5 & S. R. & EY \\
\hline CIA HERING & 29.0 & S. R. & Deloitte & 29.4 & S. R. & Deloitte & 32.5 & S. R. & KPMG \\
\hline HYPERA & 29.1 & S. R. & KPMG & 30.5 & S. R. & KPMG & 30.2 & S. R. & KPMG \\
\hline IGUATEMI & 29.5 & S. R. & KPMG & 30.6 & S. R. & KPMG & 30.0 & S. R. & EY \\
\hline ITAUSA & 27.5 & S. R. & BDO BR & 27.4 & S. R. & BDO BR & 25.0 & S. R. & BDO BR \\
\hline ITAUUNIBANCO & 27.4 & S. R. & PWC & 26.1 & S. R. & PWC & 26.5 & S. R. & PWC \\
\hline JBS & 30.1 & S. R. & BDO BR & 29.6 & S. R. & BDO BR & 27.4 & S. R. & BDO BR \\
\hline KLABIN S/A & 27.6 & S. R. & PWC & 30.1 & S. R. & PWC & 29.3 & S. R. & $\mathrm{EY}$ \\
\hline KROTON & 28.2 & S. R. & PWC & 29.2 & S. R. & PWC & 29.1 & S. R. & PWC \\
\hline LOJAS AMERIC & 28.4 & S. R. & PWC & 29.8 & S. R. & KPMG & 28.6 & S. R. & KPMG \\
\hline LIGHT S/A & 24.8 & S. R. & Deloitte & 26.8 & S. R. & Deloitte & 28.2 & S. R. & EY \\
\hline LINX & 30.5 & S. R. & KPMG & 29.5 & S. R. & KPMG & 29.4 & S. R. & EY \\
\hline LOJAS RENNER & 29.9 & S. R. & KPMG & 30.6 & S. R. & KPMG & 30.7 & S. R. & KPMG \\
\hline M.DIASBRANCO & 26.6 & S. R. & $\mathrm{EY}$ & 27.6 & S. R. & PWC & 28.1 & S. R. & PWC \\
\hline MAGAZ LUIZA & 27.1 & S. R. & EY & 28.9 & S. R. & $\mathrm{EY}$ & 31.8 & S. R. & KPMG \\
\hline MULTIPLUS & 28.7 & S. R. & KPMG & 27.2 & S. R. & EY & 27.8 & S. R. & EY \\
\hline MARFRIG & 28.1 & S. R. & BDO BR & 26.8 & S. R. & BDO BR & 29.1 & S. R. & $\begin{array}{c}\text { Grant } \\
\text { Thornton } \\
\text { Br. }\end{array}$ \\
\hline MRV & 28.8 & S. R. & EY & 28.2 & S. R. & EY & 30.7 & S. R. & KPMG \\
\hline MULTIPLAN & 30.3 & S. R. & KPMG & 31.0 & S. R. & KPMG & 31.0 & S. R. & KPMG \\
\hline IOCHP-MAXION & 29.7 & S. R. & Deloitte & 28.5 & S. R. & Deloitte & 30.6 & S. R. & Deloitte \\
\hline NATURA & 28.3 & S. R. & EY & 30.0 & S. R. & EY & 29.6 & S. R. & KPMG \\
\hline ODONTOPREV & 29.3 & S. R. & KPMG & 28.9 & S. R. & KPMG & 29.8 & S. R. & KPMG \\
\hline P.ACUCAR-CBD & 32.4 & S. R. & Deloitte & 28.3 & S. R. & Deloitte & 29.5 & S. R. & EY \\
\hline PETROBRAS & 25.6 & S. R. & PWC & 26.9 & S. R. & PWC & 30.4 & S. R. & KPMG \\
\hline MARCOPOLO & 30.0 & S. R. & KPMG & 32.3 & S. R. & KPMG & 29.4 & S. R. & PWC \\
\hline
\end{tabular}




\begin{tabular}{|c|c|c|c|c|c|c|c|c|c|}
\hline PORTO SEGURO & 29.7 & S. R. & Deloitte & 27.4 & S. R. & Deloitte & 27.2 & S. R. & PWC \\
\hline QUALICORP & 29.7 & S. R. & Deloitte & 27.7 & S. R. & PWC & 28.3 & S. R. & PWC \\
\hline RAIADROGASIL & 28.0 & S. R. & PWC & 27.9 & S. R. & PWC & 28.0 & S. R. & PWC \\
\hline RUMO S.A. & 30.3 & S. R. & KPMG & 29.7 & S. R. & KPMG & 31.3 & S. R. & KPMG \\
\hline RANDON PART & 30.1 & S. R. & KPMG & 28.8 & S. R. & KPMG & 29.1 & S. R. & KPMG \\
\hline LOCALIZA & 27.9 & S. R. & PWC & 29.4 & S. R. & PWC & 29.9 & S. R. & Deloitte \\
\hline SANTANDER BR & 26.3 & S. R. & Deloitte & 27.8 & S. R. & PWC & 27.4 & S. R. & PWC \\
\hline SANEPAR & 27.6 & S. R. & EY & 29.4 & S. R. & EY & 29.9 & S. R. & $\begin{array}{l}\text { BDO } \\
\text { Brazil }\end{array}$ \\
\hline SABESP & 35.4 & S. R. & Deloitte & 31.8 & S. R. & KPMG & 30.6 & S. R. & KPMG \\
\hline SER EDUCA & 29.6 & S. R. & PWC & 30.8 & S. R. & PWC & 30.0 & S. R. & PWC \\
\hline SÃO MARTINHO & 28.3 & S. R. & EY & 27.7 & S. R. & EY & 28.1 & S. R. & EY \\
\hline SUL AMÉRICA & 29.1 & S. R. & Deloitte & 29.0 & S. R. & Deloitte & 29.1 & S. R. & Deloitte \\
\hline SUZANO PAPEL & 30.1 & S. R. & KPMG & 32.1 & S. R. & KPMG & 29.5 & S. R. & PWC \\
\hline TAESA & 28.9 & S. R. & Deloitte & 30.3 & S. R. & Deloitte & 28.8 & S. R. & EY \\
\hline AES TIETE E & 30.3 & S. R. & EY & 28.9 & S. R. & EY & 28.8 & S. R. & $\mathrm{EY}$ \\
\hline TIM PART S/A & 26.8 & S. R. & Baker Tilly & 28.5 & S. R. & BDO BR & 29.0 & S. R. & BDO BR \\
\hline TOTVS & 28.1 & S. R. & PWC & 29.4 & S. R. & PWC & 29.8 & S. R. & EY \\
\hline TRAN PAULIST & 26.5 & S. R. & $\begin{array}{c}\text { Grant } \\
\text { Thornton }\end{array}$ & 26.1 & S. R. & EY & 27.2 & S. R. & EY \\
\hline ULTRAPAR & 29.8 & S. R. & Deloitte & 30.0 & S. R. & Deloitte & 30.6 & S. R. & KPMG \\
\hline USIMINAS & 32.3 & S. R. & KPMG & 32.9 & S. R. & KPMG & 29.0 & S. R. & PWC \\
\hline VALE & 25.4 & S. R. & KPMG & 31.0 & S. R. & KPMG & 31.5 & S. R. & KPMG \\
\hline TELEF BRASIL & 28.1 & S. R. & EY & 30.1 & S. R. & EY & 29.6 & S. R. & PWC \\
\hline VALID & 29.0 & S. R. & Deloitte & 28.1 & S. R. & Deloitte & 28.8 & S. R. & EY \\
\hline VIAVAREJO & 28.3 & S. R. & Deloitte & 28.4 & S. R. & Deloitte & 30.3 & S. R. & Deloitte \\
\hline WEG & 28.8 & S. R. & KPMG & 30.3 & S. R. & KPMG & 30.5 & S. R. & KPMG \\
\hline WIZ S.A. & 28.0 & S. R. & PWC & 29.1 & S. R. & PWC & 28.7 & S. R. & PWC \\
\hline
\end{tabular}

Nota: *S. R. (Sem Ressalva), C. R. (Com Ressalva).

Fonte: Dados da pesquisa.

De acordo com o exposto na Tabela 1, percebe-se que, no momento de transição de uma empresa de auditoria para outra, também houve, em alguns casos, alteração do grau de legibilidade, e, em outros, o grau de complexidade permaneceu basicamente o mesmo. Entre os anos de 2015 e 2016, a empresa AREZZO CO fazia auditoria, em 2015, com a empresa EY e, no ano de 2016, passou a fazer auditoria com a PWC. Nesse caso, observa-se que, no ano de 2015, o relatório do auditor apresentou um grau de legibilidade de 28.45 (48\% de complexidade acima do destacado na literatura, 15 graus), sofrendo, nos anos de 2016 e 2017, pequenas alterações para 28.60 e 28.69 respectivamente. Assim, não se pode afirmar que o grau de legibilidade está associado ao perfil de escrita dos relatórios das empresas de auditoria, já que essas variações de grau de complexidade (conforme Tabela 1), também ocorreram, em situação idêntica de rodízio, em quase todas as 
empresas.

Outra observação interessante foi que a empresa COPASA apresentou, no ano de 2015, um relatório de auditoria 51\% mais complexo. No ano de 2016, esse percentual de complexidade diminuiu para $48 \% \mathrm{e}$ aumentou para 49\% no ano de 2017 (ano posterior à adoção das novas normas). Apesar disso, a mesma empresa teve parecer com ressalvas nos anos de 2015 e 2016. Nesse sentido, observa-se que a alteração da norma não atingiu o seu objetivo de permitir que os auditores apresentem mais informações, de forma mais claras e objetivas, facilitando a compreensão destas por parte de seus usuários internos e externos. De acordo com a Tabela 1, todas as empresas apresentaram baixo nível de legibilidade no ano de 2015 (ano anterior à adoção das normas), assim como no ano de 2016 e em 2017.

A empresa LIGHT S/A foi a que apresentou o menor grau de complexidade de todas as empresas no ano de 2015. Entretanto, o grau de complexidade, que já era alto no ano de 2015 (41\% acima do considerado informação complexa), aumentou ainda mais em 2017, ano posterior à adoção das normas, isto é, o percentual foi para $47 \%$. Esse resultado pode estar relacionado ao perfil da empresa, em função de pertencer ao setor com regulação própria, como é o caso das empresas pertencentes ao setor de energia elétrica.

A companhia que apresentou o maior grau de complexidade no ano de 2015 foi a B2W DIGITAL, com um relatório aproximado de $147 \%$ de grau de complexidade acima do que são consideradas informações complexas (15 graus). Essa empresa, ainda, reapresentou relatos financeiros, no ano de 2015, pelo motivo: "Melhor apresentação das Notas Explicativas e Quadros". Contudo, tanto o primeiro relatório quanto o reapresentado indicaram grau de complexidade aproximado de 37 pontos. Apesar de esse percentual ter reduzido para aproximadamente $111 \%$, de 2015 para 2017, ele ainda é considerado alto. Cabe, também, destacar que a empresa B2W DIGITAL fez rodízio de empresa de auditoria. No ano de 2015, era auditada pela PWC, passando a ser auditada pela KPMG, nos anos de 2016 e 2017. Nesse sentido, avalia-se que fosse importante investigar se, naquele momento de transição (rodízio), havia alguma informação nos parágrafos de ênfase, no ano de 2015, que foi atendida pela empresa auditada e que foi observada e validada pela nova empresa de auditoria, conferindo que esse fato possa ter contribuído para a diminuição do grau de complexidade.

Na Tabela 2, apresentam-se as médias de legibilidade por empresa de auditoria, percebendo-se que a empresa de auditoria KPMG apresentou um relatório de auditoria com um grau de legibilidade $49 \%$ acima do nível considerado como informação complexa (15 graus) no ano de 2015. Esse percentual aumentou no ano (2016) em que foram estabelecidas as principais alterações nos relatórios do auditor independente, quando da adoção obrigatória das NBC TA 570, 700, 701, 705, 706 (2016). Além disso, esse grau de complexidade ficou ainda maior no ano de 2017, ano subsequente à adoção, chegando a 51\%. Os resultados também não permitiram verificar se havia uma relação entre o tipo de parecer e o grau de legibilidade dos relatórios do auditor, pois menos de $1,2 \%$ dos 273 relatórios analisados foram identificados com o tipo de parecer com ressalva. 
Tabela 2 - Médias de legibilidade por ano e por empresas de auditoria.

\begin{tabular}{c|c|c|c}
\hline \multirow{2}{*}{ Empresas de auditoria } & \multicolumn{3}{|c}{ Média FOG Ano } \\
\cline { 2 - 4 } & $\mathbf{2 0 1 5}$ & $\mathbf{2 0 1 6}$ & $\mathbf{2 0 1 7}$ \\
\hline Deloitte & 29.27 & 29.12 & 29.58 \\
\hline KPMG & 28.98 & 30.40 & 30.52 \\
\hline PWC & 28.95 & 28.6 & 28.55 \\
\hline EY & 28.17 & 28.28 & 28.76 \\
\hline Grant Thornton & 26.46 & 27.87 & 29.57 \\
\hline BDO BR & 28.28 & 31.00 & 28.06 \\
\hline Baker Tilly & 28.58 & 0 & 0 \\
\hline Todas as empresas de auditoria & 28.38 & 25.04 & 25.00 \\
\hline
\end{tabular}

Fonte: Dados da pesquisa.

Destaca-se, ainda, que a média de todas as empresas teve uma redução de $48 \%$ para $40 \%$ no grau de complexidade, tanto no ano de 2016 como em 2017. Quanto ao grau de legibilidade, entretanto, as empresas DELOITTE e EY foram as únicas que não tiveram alterações bruscas. Cabe destacar que, apesar dessas constatações, todas as empresas apresentaram grau de complexidade superior ao considerado "nível complexo de entendimento" da informação divulgada (15 pontos). Se analisado de forma geral, pode-se inferir que, no geral, todas as empresas de auditoria apresentaram relatórios de auditoria mais complexos, tanto antes quanto depois da adoção das normas. É possível, nesse sentido, que uma análise mais profunda identificasse se o fator de alteração do grau de complexidade foram as características de exigências de cada norma, ou se, simplesmente, como forma de elevar o grau de compreensão das informações nos relatórios estes apresentaram informações mais complexas. De qualquer modo, por esta análise, em ambos os sentidos, a mudança dos relatórios pode até ter melhorado ou simplificado alguns pontos; entretanto, as informações ficaram mais difíceis de serem lidas.

Na Figura 1, demonstram-se, por meio de quatro histogramas, quais foram os níveis de distribuição do grau de legibilidade e respectivos níveis de governança. O histograma “0”, refere-se às empresas com os papéis mais líquidos do Índice Brasil 100 (IBrX 100), mas que não possuem nível de governança. No histograma “1”, relacionam-se as empresas que estão no Nível 1 de Governança Corporativa; no histograma "2", as empresas que pertencem ao Nível 2 de Governança Corporativa; e, por fim, o histograma 3 tem por base as empresas que pertencem ao Nível de Governança Corporativa Novo Mercado. 
Figura 1 - Histogramas por níveis de governança.
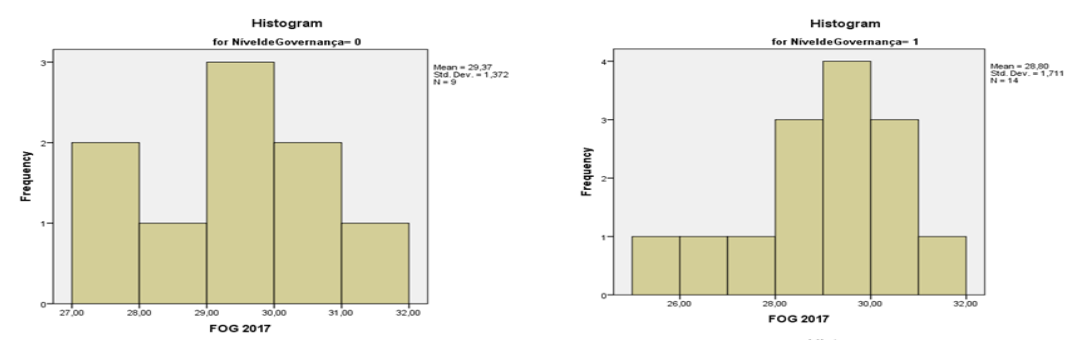

Histogram Histogram
tor Nivelde Governans
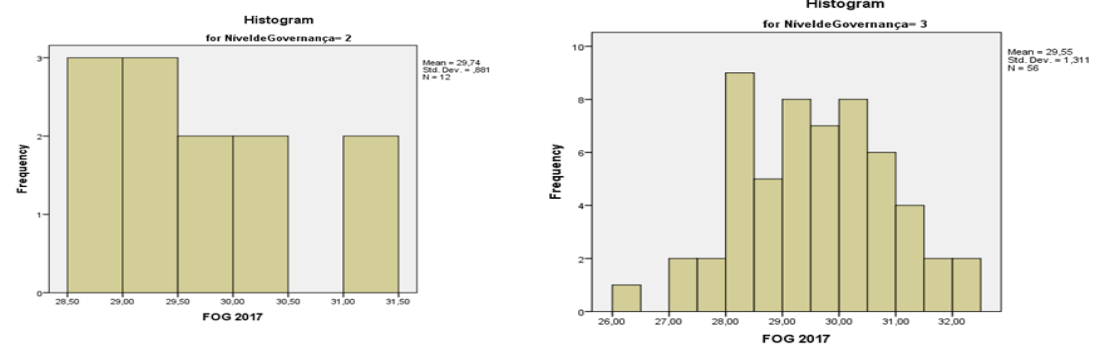

Fonte: Dados da pesquisa.

Conforme representado na Figura 1, percebe-se que o nível de variação do índice FOG ocorreu nos três níveis de governança, assim como naquelas empresas que não estão em nível de governança nenhum. Entretanto, observa-se que, no Nível 2 de governança, o índice de legibilidade está mais próximo de uma distribuição das empresas sem nível de governança. Quanto ao Nível 1 de governança, o índice de legibilidade se concentra mais à direita; enquanto, no nível Novo Mercado, essa distribuição tende à centralidade.

Em relação à normalidade dos dados, ilustrada na Figura 2 (Gráficos QQ), a seguir, mostra-se o teste dos dados relativos aos índices FOG e aos níveis de Governança Corporativa, QQ plot (quantil-quantil). Segundo Fávero et al. (2017), o QQ plot pode ser entendido como um método gráfico de probabilidades usado para comparar formas de distribuição.

Figura 2 - Distribuição da normalidade dos FOG.
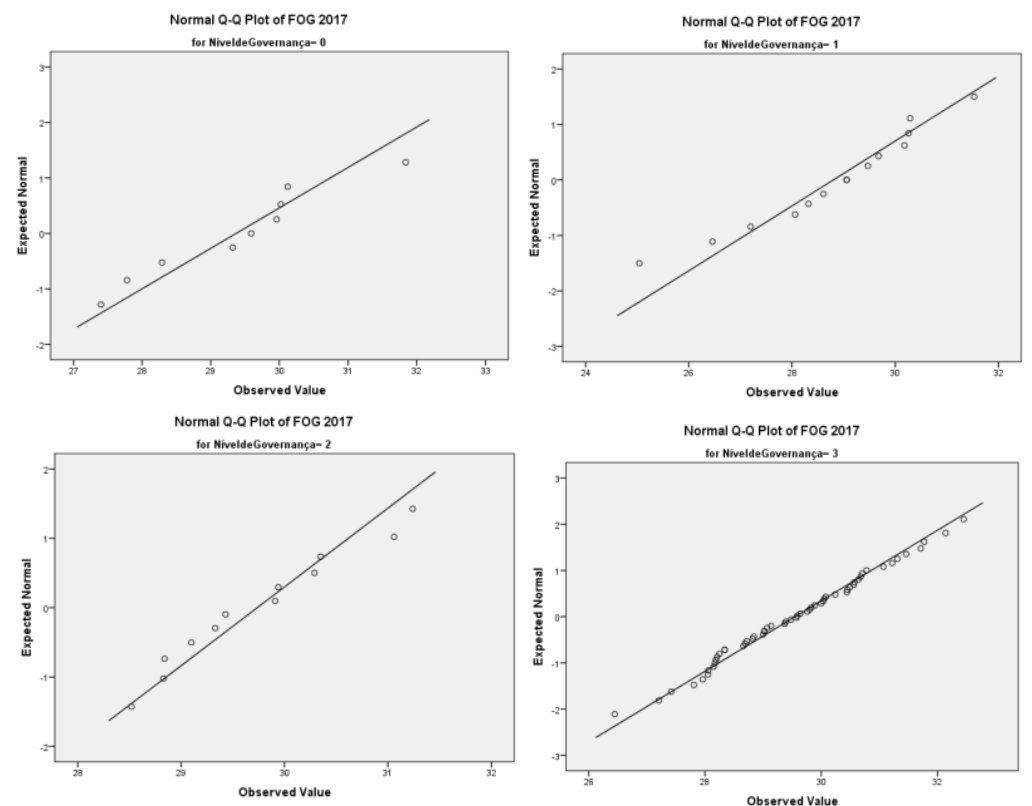

Fonte: Dados da pesquisa. 
Para a variável FOG, conforme se observa, a partir da Figura 2 (gráficos Q-Q), não se pode afirmar que os dados seguem uma distribuição normal, uma vez que apresenta uma variação da cauda em referência à linha, simulando a distribuição normal para a distribuição do índice FOG em grupos de empresas sem Governança Corporativa e empresas pertencentes aos níveis 1 e 2 com Governança Corporativa. Entretanto, conforme o Q-Q no nível de Novo Mercado, há uma aproximação da cauda de referência, que simula a normalidade dos índices FOG nesse nível de governança.

Na Tabela 3, a seguir, onde se representa o percentual de participação de cada empresa com e sem nível de governança, observa-se que mais de $50 \%$ das empresas com os papéis mais líquidos, listados no Índice Brasil 100 (IBrX 100), estão vinculadas ao nível de governança Novo Mercado. Apesar disso, nota-se que a média dos índices de legibilidade variou, de forma muito próxima, entre as empresas com e sem nível de governança, ou seja, as empresas sem nível de governança apresentaram uma média de índice de legibilidade de 29,37.

As empresas do nível 1 de governança apresentaram uma média de índice FOG de 28,80. As empresas com nível 2 de governança tiveram uma média de 29,73 no índice de legibilidade e, por fim, as empresas do Novo Mercado apresentaram uma média de índice FOG de 29,55. Diante disso, em princípio, não é possível afirmar que, em função de seus níveis, empresas com mais atributos de governança corporativa apresentam informações menos complexas e, portanto, "menos difíceis" de serem compreendidas.

Tabela 3: Distribuição das empresas por nível de governança.

\begin{tabular}{|c|c|c|c|c|c|c|c|}
\hline \multirow{2}{*}{\multicolumn{2}{|c|}{ Nível de Governança }} & \multicolumn{6}{|c|}{ Resultados } \\
\hline & & $\mathrm{N}$ & $\%$ & $\mathrm{~N}$ & $\%$ & $\mathrm{~N}$ & $\%$ \\
\hline \multirow{4}{*}{ FOG 2017} & 0 & 9 & $9,89 \%$ & 0 & $0.0 \%$ & 9 & $100.0 \%$ \\
\hline & 1 & 14 & $15,38 \%$ & 0 & $0.0 \%$ & 14 & $100.0 \%$ \\
\hline & 2 & 12 & $13,19 \%$ & 0 & $0.0 \%$ & 12 & $100.0 \%$ \\
\hline & 3 & 56 & $61,54 \%$ & 0 & $0.0 \%$ & 56 & $100.0 \%$ \\
\hline
\end{tabular}

Fonte: Dados da pesquisa.

Na sequência, a Figura 3 (boxplot dos anos de 2015 a 2017) expõe o diagrama que representa a posição, dispersão, simetria e os valores discrepantes (outliers) do conjunto dos $273 \mathrm{FOG}$, analisados neste estudo, por ano.

Figura 3 - Boxplot.

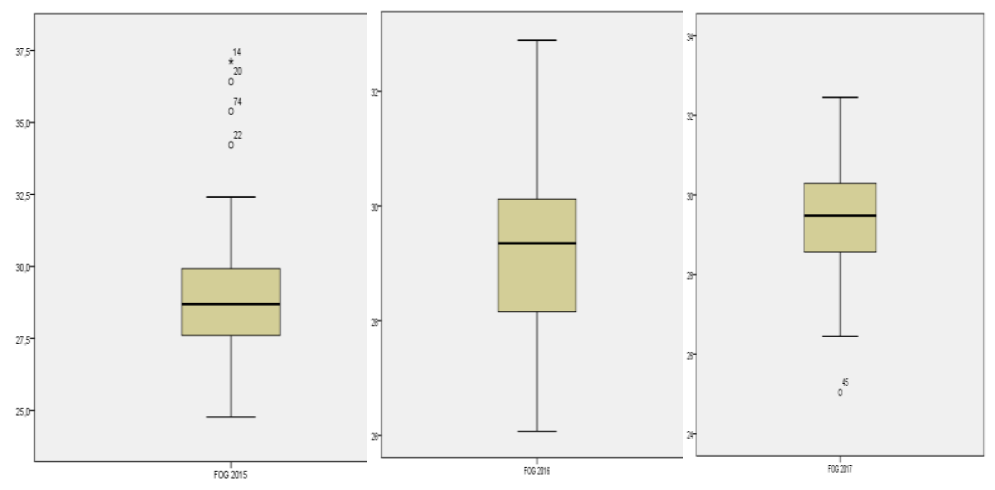

Fonte: Dados da pesquisa.

Analisando exposto na Figura 3, observa-se que o ano de 2015 foi o que mais apresentou discrepância 
de índice de legibilidade. Em relação à simetria dos FOG, no ano de 2015, a linha mediana do segundo quartil esteve muito próxima do centro do boxplot. Entretanto, no ano de 2017, a simetria dos FOG foi ainda maior, ou seja, aquela diferença (outliers), observada no ano anterior à mudança das normas (2015), basicamente, desaparece. Isso significa que o perfil do grau de legibilidade ficou muito mais simétrico no ano de 2017 (ano posterior à mudança das normas), demonstrando que, apesar de o grau de complexidade ainda ser alto, há menor discrepância entre os relatórios apresentados pelas empresas de auditoria. Desse modo, sugere-se que, em nível de divulgação de informações padronizadas, a mudança nas normas dos relatórios tem alcançado o seu propósito. Cabe destacar, portanto, que a diferença maior no intervalo interquartílico se deu apenas no ano de 2016, mesmo ano em que também não se observou outliers interferindo nessa tendência. Tal resultado pode ser explicado em função dos ajustes necessários, feitos pelas empresas de auditoria em seus relatórios de auditoria, já que as mudanças nas normas para elaboração do novo relatório passaram a vigorar em 2016.

Por fim, para verificar se existe correlação entre o grau de legibilidade dos relatórios do auditor independente e o fato de as empresas pertencerem ou não a algum nível de Governança Corporativa, ou seja, medir a força de associação entre essas variáveis, realizou-se o teste estatístico de correlação de Spearman (Tabela 4). Para a realização desse teste, as empresas que não possuíam nível de governança receberam " 0 ", enquanto as empresas com níveis 1 e 2 de governança e Novo Mercado receberam " 1 ".

Tabela 4: Estimativas de correlação de Spearman.

\begin{tabular}{ccc}
\hline & & Peso Estrato \\
\hline \multirow{3}{*}{ FOG } & Coeficiente de Correlação & 0,777 \\
& Valor-p & 0,030 \\
& $\mathrm{~N}$ & 91 \\
\hline
\end{tabular}

Fonte: Dados da pesquisa.

Segundo Fávero et al. (2017), o coeficiente de correlação de Spearman mede a força de associação entre variáveis. Nesta análise, como o coeficiente de correlação de Spearman ( $\rho$ ) foi de 0,777, pode-se entender que existe uma correlação positiva e com magnitude moderada entre as variáveis grau de legibilidade com o fato de as empresas pertencerem a níveis de Governança Corporativa. Além disso, conforme se demonstrou na Figura 1, observa-se maior tendência dos níveis de complexidade em empresas do Nível 1 de Governança Corporativa; enquanto, no nível de governança Novo Mercado, observa-se uma padronização do nível de complexidade, sugerindo que, apesar da correlação positiva entre o grau de complexidade e os níveis de governança, maiores níveis de governança (níveis com maiores exigências de requisito de governança), nem sempre resultam em divulgações menos complexas.

\section{Considerações Finais}

O presente estudo teve como objetivo analisar se o grau de legibilidade dos relatórios do auditor independente, entre os anos de 2015 e 2017, foi afetado após as mudanças das normas de elaboração desses relatórios. Para tanto, foram analisados os pareceres das empresas com as ações listadas no Índice Brasil 100 (IBrX 100), disponíveis no site da [B] $]^{3}$ Brasil, Bolsa, Balcão.

O nível de legibilidade entre esses anos, mesmo com a mudança, se manteve alto em relação ao nível considerado "compreensível", mostrando, dessa forma, que essa mudança pouco influiu, positivamente, no 
grau de legibilidade desses relatórios. Na empresa VALE S.A., por exemplo, os índices de legibilidade aumentaram, consideravelmente, em 2016, ano da adoção da mudança, e tornou a crescer em 2017. Nas companhias que apresentaram uma diminuição neste índice, essa variação, mesmo que positiva (contribuindo para uma melhor compreensão do documento), ainda esteve acima do nível ideal do índice de legibilidade.

Considerando a relevância da legibilidade da informação financeira, destacada no decorrer deste estudo, os resultados obtidos na pesquisa podem indicar desde uma maior dificuldade em se investir no Brasil, levando em conta os pequenos investidores, pela falta de compreensão da informação, até tomadas de decisão menos conscientes por parte desses investidores. Assim, infere-se que o ambiente de investigações pode contribuir para a divulgação de informações mais complexas (JONES, 1996; BUSCHEE; GOW; TAYLOR, 2018; LEHAVY; LI; MERKLEY, 2011; COURTIS, 1986; COURTIS, 1995).

Entende-se que esta pesquisa apresenta como limitação a análise geral do texto e de como o grau de legibilidade foi afetado pelas novas mudanças nas normas do Relatório do Auditor Independente. Futuras pesquisas poderiam examinar mais profundamente a alteração do grau de legibilidade por tópicos específicos. Ou seja, como o objetivo do novo relatório do auditor é fazer com que o auditor apresente informações, que sejam mais claras e objetivas para facilitar a compreensão dos usuários internos e externos, seria muito útil verificar como o grau de legibilidade foi afetado em cada uma das principais alterações (partes do relatório do auditor), a saber: NBC TA 570 (responsabilidade do auditor de encontrar evidências); NBC TA 700 (a opinião do auditor, que passa a ser publicada no início do relatório); NBC TA 701 (a responsabilidade do auditor de comunicar os principais assuntos de auditoria no relatório); NBC TA 705 (opinião do auditor que pode se apresentar como: opinião limpa, opinião com ressalva ou opinião adversa, além de poder se abster de opinar); e NBC TA 706 (permite ao auditor escrever Parágrafos de Ênfase no relatório).

\section{Referências}

BAKER, E., KARE, D. D. Relationship between annual report readability and corporate financial performance. Management Research News, v. 15, n. 1, p. 1-4, 1992. DOI: https://doi.org/10.1108/eb028188

BUSCHEE, B. J.; GOW, I. D.; TAYLOR, D. J. Linguistic Complexity in Firm Disclosures: Obfuscation or Information? Journal of Accounting Research, v. 56, n. 1, p. 85-121, 2018. DOI: https://doi.org/10.1111/1475-679X.12179

CONSELHO FEDERAL DE CONTABILIDADE (CFC). Resolução CFC n. 1.231/09. NBC TA 700 - Formação da Opinião e Emissão do Relatório do Auditor Independente sobre as Demonstrações Contábeis. Disponível em: http://www2.cfc.org.br/sisweb/sre/detalhes_sre.aspx?codigo=2016/NBCTA700.

COURTIS, J. K. An Investigation into Annual Report Readability and Corporate Risk-Return Relationships. Accounting and Business Research, v. 16, n. 64, p. 284-294, 1986. DOI: https://doi.org/10.1080/00014788.1986.9729329.

Readability of annual reports: Western versus Asian evidence. Accounting, Auditing and Accountability Journal, v. 8 n. 2, p. 4-17, 1995. DOI: https://doi.org/10.1108/09513579510086795.

CURTO, P. S. L. Classificador de textos para o ensino de português como segunda língua. 2014. 132 f. Dissertação (Mestrado em Engenharia) - Universidade de Lisboa, Lisboa, Portugal, 2014. 
Demonstrações financeiras Exercício de 2013. O aperfeiçoamento das regras contábeis brasileiras. Disponível em: https://www.iasplus.com/en/publications/brazil/other/guia2013/at_download/file/Guia\%202013.pdf Acesso em: 18 de jul. 2017.

FÁVERO, L. P.; BELFIORE, P.; TAKAMATSU, R. T.; SUZART, J. Métodos Quantitativos com Stata: Procedimentos, Rotinas e Análise de Resultados. 1. ed. Rio de Janeiro: Elsevier, 2017.

FAKHFAKH, M. The readability of international illustration of auditor's report: an advanced reflection on the compromise between normative principles and linguistic requirements. Journal of Economics, Finance and Administrative Science, v. 20, n. 38, p. 21-29, 2015. DOI: https://doi.org/10.1016/j.jefas.2015.02.001.

GUNNING, R. The Technique of Clear Writing. McGraw-Hill. p. 36-37, 1952.

IAASB divulga proposta para novo relatório do auditor. IBRACON. Disponível em: http://www.ibracon.com.br/ibracon/Portugues/detNoticia.php?cod=1313. Acesso em: 18 de jul. 2017.

LEHAVY, R.; LI, F.; MERKLEY, K. The Effect of Annual Report Readability on Analyst Following and the Properties of Their Earnings Forecasts. The Accounting Review. v. 86, n. 3 pp. 1087-1115, 2011. DOI: https://doi.org/10.2308/accr.00000043

LI, F. Annual report readability, current earnings, and persistence. Journal Accounting and Economics, v. 45, n. 2, p. 221-247, 2008. DOI: https://doi.org/10.1016/j.jacceco.2008.02.003

LO, K.; RAMOS, F.; ROGO, R. Earnings Management and Annual Report Readability. Journal of Accounting and Economics, v. 63, n. 1, p. 1-25, 2017. DOI: https://doi.org/10.1016/j.jacceco.2016.09.002

Normas do Novo Relatório do Auditor Independente. CFC. Disponível em: http://cfc.org.br/noticias/normas-donovo-relatorio-do-auditor-independente-sao-publicadas-pelo-cfc/. Acesso em: 20 de jul. 2017.

$[\mathrm{B}]^{3}$ BRASIL, BOLSA, BALCÃO. Seguimentos de listagem: Novo Mercado. Disponível em: http://www.bmfbovespa.com.br/pt_br/listagem/acoes/segmentos-de-listagem/novo-mercado Acesso em: 15 de dez. 2017.

MILLER, B. P. The Effects of Reporting Complexity on Small and Large Investor Trading. The Accounting Review, v. 85, n. 6, p. 2107-2143, 2010. DOI: https://www.jstor.org/stable/i27895901

MIRANDA, I. A.; REINA, D.; LEMES, S. Grau de legibilidade dos relatórios financeiros em empresas do novo mercado. In: XVIII USP International Conference in Accounting: Moving Accounting Forward. Anais... São Paulo: 2018.

MORENO, A.; CASASOLA, A. A readability evolution of narratives in annual reports: A longitudinal study of two Spanish companies. Journal of Business and Technical Communication, v. 30, n. 2, p. 202-235. 2016. https://doi.org/10.1177/1050651915620233

PATROCINIO, E. A.; COUTINHO, R. S.; REINA, D. R. M.; REINA, D. Pareceres de auditoria nas empresas do novo mercado: principais motivos de ressalvas e de parágrafos de ênfase. In: CONGRESSO UFU DE CONTABILIDADE, 2., 2017, Uberlândia. Anais eletrônicos... Uberlândia: UFU, 2017.

PORTO, J. S.; PAIVA, T. S. S.; AMARAL, C. L. F.; REBOUÇAS, T. N. H.; SILVA, R. A. Legibilidade de artigos de um periódico nacional na área do melhoramento vegetal. Cultivando o Saber, v. 7, n. 2, p. 205-211, 2014. 
RICCI, A. A. R. V. Análise do conteúdo aplicada aos relatórios de auditoria das empresas de tecnologia da informação no Brasil. 2014. 88 f. Dissertação (Mestrado em Ciências Contábeis). Programa de PósGraduação em Ciências Contábeis. Fundação Escola de Comércio Álvares Penteado (FECAP). São Paulo, 2014.

RENNEKAMP, K. Processing Fluency and Investors Reactions to Disclosure Readability. Journal of Accounting Research, v. n. 50 p. 1319-1354, 2012. https://doi.org/10.1111/j.1475-679X.2012.00460.x

SANTOS, E. S., CALIXTO, L., BISPO, M. F. Impacto da OCPC 07 no enxugamento das notas explicativas das companhias brasileiras. Revista Contabilidade e Finanças, v. 30, n. 79, p. 58-72, 2018. DOI: $\underline{\text { https://doi.org/10.1590/1808-057x201806650 }}$

SILVA, G. D. M.; LEMES, S.; REINA, D. Notas explicativas sob a ótica da OCPC 07: um estudo em empresas do segmento IBRX-50. Revista de Administração, Contabilidade e Economia. V. 11, n. 3, p. 36-55, 2020. DOI: HTTP://DX.DOI.ORG/10.13059/RACEF.V11I3.620

SILVA, C. A. T.; FERNANDES, J. L. T. Legibilidade dos fatos relevantes no Brasil. RAC-Electronica, v. 3, n. 1, p. 142-159, 2009.

SOUZA, J. A. S.; RISSATTI, J. C.; ROVER, S.; BORBA, J. A. The linguistic complexities of narrative accounting disclosure on financial statements: An analysis based on readability Characteristics. Research in International Business and Finance, v. 48, p 59-74, 2019. DOI: https://doi.org/10.1016/j.ribaf.2018.12.008 